\title{
A VARIATIONAL McSHANE INTEGRAL CHARACTERISATION OF THE WEAK RADON-NIKODYM PROPERTY
}

\author{
SOKOL BUSH KALIAJ
}

(Received 23 June 2011)

Abstract

We present a characterisation of Banach spaces possessing the weak Radon-Nikodym property in terms of finitely additive interval functions whose McShane variational measures are absolutely continuous with respect to Lebesgue measure.

2010 Mathematics subject classification: primary 28B05; secondary 46G10.

Keywords and phrases: weak Radon-Nikodym property, Banach space, finitely additive interval function, McShane variational measure.

\section{Introduction}

In [1], Bongiorno et al. have shown characterisations of Banach spaces possessing the weak Radon-Nikodym property (WRNP) in terms of finitely additive interval functions. They proved that a Banach space $X$ has the WRNP if and only if, for every $X$-valued finitely additive interval function $\varphi$ that has absolutely continuous Henstock variational measure, there is a Henstock-Kurzweil-Pettis integrable function $f:[0,1] \rightarrow X$ such that

$$
\varphi(I)=(\mathrm{HKP}) \int_{I} f \quad \text { for every interval } I \subset[0,1],
$$

where (HKP) $\int_{I} f$ denotes the Henstock-Kurzweil-Pettis integral of $f$ over $I$; see [1, Definition 2.2].

In this paper, we present a characterisation of Banach spaces possessing the WRNP in terms of finitely additive interval functions whose McShane variational measures are absolutely continuous with respect to Lebesgue measure. We prove that a Banach space $X$ has the WRNP if and only if, for every $X$-valued finitely additive interval function $\varphi$ that has absolutely continuous McShane variational measure, there is a weakly McShane integrable function $f:[0,1] \rightarrow X$ such that (1.1) holds true for every interval $I \subset[0,1]$ (but now the integral is the weak McShane integral).

(c) 2012 Australian Mathematical Publishing Association Inc. 0004-9727/2012 \$16.00 
Henstock and McShane variational measures have been used extensively for studying the primitives (indefinite integrals) of real functions. See, for example, the papers by Di Piazza [3] and Lee [4] and the book Pfeffer [5] for relations to integration; see also the fundamental general work by Thomson [10].

\section{Basic definitions}

Throughout this paper, $X$ denotes a real Banach space with its norm $\|\cdot\|$. By $X^{*}$ we denote the dual to $X$. Given a functional $x^{*} \in X^{*}$ its value on the element $x \in X$ will be denoted by $x^{*}(x)$.

Let $S$ be the unit interval $[0,1]$ of the real line equipped with the usual topology and the Lebesgue measure $\lambda$. We denote by $\mathcal{L}$ the family of all Lebesgue measurable subsets of $S$ and by $\mathscr{S}$ the family of all nondegenerate closed subintervals of $S$. The intervals $I$ and $J$ are said to be nonoverlapping if $\operatorname{int}(I) \cap \operatorname{int}(J)=\emptyset$, where $\operatorname{int}(I)$ denotes the interior of $I$.

A mapping $v: \mathcal{L} \rightarrow X$ is said to be an $X$-valued measure if $v$ is countable additive in the norm topology of $X$. An $X$-valued measure is said to be $\lambda$-continuous if $\lambda(E)=0$ implies $v(E)=0$. The variation of an $X$-valued measure $v$ is denoted by $|v|$.

A function $\varphi: \mathscr{S} \rightarrow X$ is said to be an interval function. An interval function $\varphi: \mathscr{S} \rightarrow X$ is said to be finitely additive if $\varphi(I \cup J)=\varphi(I)+\varphi(J)$ for all nonoverlapping intervals $I, J \in \mathscr{S}$ with $I \cup J \in \mathscr{S}$. We denote by $\Phi$ the family of all finitely additive interval functions $\varphi: \mathscr{S} \rightarrow X$

A function $\varphi \in \Phi$ is said to be strongly absolutely continuous (or briefly $s A C$ ) if for every $\varepsilon>0$ there exists $\eta>0$ such that, for every finite collection $\left\{I_{i}: i=1,2, \ldots, n\right\}$ of nonoverlapping intervals in $\mathscr{S}$,

$$
\sum_{i=1}^{n} \lambda\left(I_{i}\right)<\eta \Rightarrow \sum_{i=1}^{n}\left\|\varphi\left(I_{i}\right)\right\|<\varepsilon .
$$

We denote by $\langle a, b\rangle$ the closed interval $[\min \{a, b\}, \max \{a, b\}], a, b \in \mathbb{R}$. A function $\varphi \in \Phi$ is said to be differentiable at $s \in S$, if there exists $x \in X$ such that

$$
\lim _{h \rightarrow 0} \frac{\varphi(\langle s, s+h\rangle)}{|h|}=x .
$$

We write $x=\varphi^{\prime}(s)$ to denote the derivative of $\varphi$ at $s$.

We say that a function $\varphi \in \Phi$ is pseudodifferentiable on $S$ if there exists a function $\varphi_{p}^{\prime}: E \rightarrow X$ such that, for every $x^{*} \in X^{*}$,

$$
\lim _{h \rightarrow 0} \frac{x^{*} \varphi(\langle s, s+h\rangle)}{|h|}=x^{*} \varphi_{p}^{\prime}(s),
$$

for almost all $s \in S$. (The exceptional sets depend on $x^{*}$.) The function $\varphi_{p}^{\prime}$ is said to be a pseudoderivative of $\varphi$. 
A pair $(I, s)$ of an interval $I \in \mathscr{S}$ and a point $s \in S$ is said to be the McShane tagged interval; $s$ is said to be the tag of $I$. Requiring $s \in I$ for the tag of $I$ we get the concept of a Henstock-Kurzweil tagged interval.

A McShane partition (or M-partition) $\pi$ in $S$ is a finite collection of McShane tagged intervals $(I, s)$ whose corresponding intervals are nonoverlapping. Similarly, a Henstock-Kurzweil partition (or $\mathcal{H} \mathcal{K}$-partition) $\pi$ in $S$ is a finite collection of Henstock-Kurzweil tagged intervals $(I, s)$ whose corresponding intervals are nonoverlapping.

A function $\delta: E \rightarrow(0,+\infty)$ is said to be a gauge on $E$, where $E$ is a subset of $S$. We say that an $\mathcal{M}$-partition $\pi$ in $S(\mathcal{H} \mathcal{K}$-partition $\pi$ in $S)$ is:

- $\quad$ an $\mathcal{M}$-partition of $S(\mathcal{H} \mathcal{K}$-partition of $S)$ if $\bigcup_{(I, s) \in \pi} I=S$;

- $\quad E$-tagged if, for all $(I, s) \in \pi, s \in E$;

- $\quad \delta$-fine, if, for every tagged interval $(I, s) \in \pi, I \subset(s-\delta(s), s+\delta(s))$.

Definition 2.1. A function $f: S \rightarrow X$ is said to be McShane integrable on $S$ and $w_{S} \in X$ is its McShane integral on $S$ if, for every $\varepsilon>0$, there exists a gauge $\delta$ on $S$ such that, for every $\delta$-fine $\mathcal{M}$-partition $\pi$ of $S$,

$$
\left\|\sum_{(I, s) \in \pi} f(s) \lambda(I)-w_{S}\right\|<\varepsilon .
$$

We write $w_{S}=(M) \int_{S} f$. A function $f: S \rightarrow X$ is said to be McShane integrable on $E \subset S$ if the function $f \cdot \chi_{E}: S \rightarrow X$ is McShane integrable on $S$, where $\chi_{E}$ is the characteristic function of the set $E$. The McShane integral of $f$ over $E$ will be denoted by $(M) \int_{E} f$. Thus we have

$$
\text { (M) } \int_{E} f=(M) \int_{S} f \cdot \chi_{E} \text {. }
$$

If $f$ is McShane integrable on $S$ then we obtain by [7, Theorem 4.1.6] that for every $E \in \mathcal{L}$ the function $f$ is McShane integrable on $E$.

Definition 2.2. We say that a function $f: S \rightarrow X$ is strongly McShane integrable (or briefly $\mathcal{S} \mathcal{M}$-integrable) on $S$ if there exists $\varphi \in \Phi$ such that, for every $\varepsilon>0$, there exists a gauge $\delta$ on $S$ such that, for every $\delta$-fine $\mathcal{M}$-partition $\pi$ of $S$,

$$
\sum_{(I, s) \in \pi}\|f(s) \lambda(I)-\varphi(I)\|<\varepsilon .
$$

By [7, Proposition 3.6.16] we obtain $\varphi(I)=(M) \int_{I} f$, for each $I \in \mathscr{S}$.

Skvortsov and Solodov defined the McShane variational integrability of functions $f: I \rightarrow X$, where $I$ is a nondegenerate compact interval of $\mathbb{R}^{m}, m \in \mathbb{N}$; see [8]. This notion coincides with $\mathcal{S} \mathcal{M}$-integrability from Definition 2.2.

If $X$ is a finite dimensional Banach space then we obtain by [7, Theorem 5.2.2] that Definitions 2.1 and 2.2 are equivalent. 
Definition 2.3. A function $f: S \rightarrow X$ is said to be weakly McShane integrable (or briefly $\mathcal{W} M$-integrable) on $S$ if, for every $x^{*} \in X^{*}$, the real function $x^{*} f$ is McShane integrable on $S$ and, for every $I \in \mathscr{S}$, there exists $w_{I} \in X$ such that (M) $\int_{I} x^{*} f=x^{*}\left(w_{I}\right)$. We call $w_{I}$ the weak McShane integral of $f$ over $I$ and we write $w_{I}=(W M) \int_{I} f$. The additive interval function $F(I)=(W M) \int_{I} f$ is said to be the WM-primitive of $f$.

According to [7, Theorem 5.2.3] a real-valued function is McShane integrable if and only if it is Lebesgue integrable. It follows that, if a function $f: S \rightarrow X$ is Pettis integrable, then the function $f$ is $\mathcal{W M}$-integrable and, for every $I \in \mathscr{S}$,

$$
\text { (P) } \int_{I} f=(W M) \int_{I} f
$$

where $(P) \int_{I} f$ denotes the Pettis integral of $f$ on $I$. In [11], Ye and Schwabik have shown that there exists a $\mathcal{W M}$-integrable function that is not Pettis integrable.

Given $\varphi \in \Phi$, a subset $E \subset S$ and gauge $\delta$ on $E$, we define

$$
V_{\varphi}^{\mathcal{M}}(E, \delta)=\sup \sum_{(I, t) \in \pi}\|\varphi(I)\|,
$$

where the supremum is taken over all $E$-tagged, $\delta$-fine, $\mathcal{M}$-partitions $\pi$ in $S$. Then we set

$$
V_{\varphi}^{\mathcal{M}}(E)=\inf \left\{V_{\varphi}^{\mathcal{M}}(E, \delta): \delta \text { is a gauge on } E\right\} .
$$

The set function $V_{\varphi}^{\mathcal{M}}$ is said to be the McShane variational measure (or $\mathcal{M}$-variational measure) generated by $\varphi$. According to Thomson's results from [9], it is known that $V_{\varphi}^{\mathcal{M}}$ is a Borel metric outer measure on $S$. We say that the McShane variational measure $V_{\varphi}^{\mathcal{M}}$ is absolutely continuous with respect to Lebesgue measure (or briefly $\left.V_{\varphi}^{\mathcal{M}}(E) \ll \lambda\right)$, if $\lambda(E)=0$ implies that $V_{\varphi}^{\mathcal{M}}(E)=0$.

If we replace $\mathcal{M}$-partitions by $\mathcal{H} \mathcal{K}$-partitions in the definition of McShane variational measure we obtain the definition of Henstock variational measure, [1, Definition 3.1]. We denote by $V_{\varphi}^{\mathcal{H}}$ the Henstock variational measure generated by $\varphi \in \Phi$.

\section{The main result}

The following lemma was proved by Di Piazza in [3, Proposition 1]. (There she considers real-valued functions, but the proof works also for vector valued functions, after trivial changes.)

Lemma 3.1. If $\varphi \in \Phi$, then $V_{\varphi}^{\mathcal{M}} \ll \lambda$ if and only if $\varphi$ is $s A C$.

We now present the main theorem.

Theorem 3.2. Let $X$ be a Banach space and let $\varphi \in \Phi$. Then the following statements are equivalent. 
(i) X has the WRNP.

(ii) If $V_{\varphi}^{\mathcal{M}} \ll \lambda$, then $\varphi$ is pseudodifferentiable on $S$.

(iii) If $V_{\varphi}^{\mathcal{M}} \ll \lambda$, then there exists a function $f: S \rightarrow X$ such that $f$ is $\mathcal{W} \mathcal{M}$-integrable on $S$ and, for every $I \in \mathscr{S}$,

$$
\varphi(I)=(W M) \int_{I} f .
$$

Proof. (i) $\Rightarrow$ (ii). Assume that $V_{\varphi}^{\mathcal{M}} \ll \lambda$. Since each $\mathcal{H} \mathcal{K}$-partition is an $\mathcal{M}$-partition, we obtain $V_{\varphi}^{\mathcal{H}} \ll \lambda$. Therefore the statement (v) of Theorem 4.5 in [1] implies that $\varphi$ is pseudodifferentiable on $S$.

(ii) $\Rightarrow$ (iii). Assume that $V_{\varphi}^{\mathcal{M}} \ll \lambda$ and let $\varphi_{p}^{\prime}$ be a pseudoderivative of $\varphi$. We will prove that the function $f=\varphi_{p}^{\prime}$ is $\mathcal{W} \mathcal{M}$-integrable with $\mathcal{W} \mathcal{M}$-primitive $\varphi$.

Assume that an arbitrary $I \in \mathscr{S}$ and an arbitrary vector $x^{*} \in X^{*}$ are given. Note that $x^{*} \varphi$ is $s A C$ and $\left(x^{*} \varphi\right)^{\prime}(s)=x^{*}(f(s))$ almost everywhere in $S$. Therefore, Theorem 7.4.13 together with [7, Theorem 5.2.2] yields that the real-valued function $x^{*} f$ is McShane integrable on $S$ with the primitive $x^{*} \varphi$. Thus,

$$
\text { (M) } \int_{I} x^{*} f=\left(x^{*} \varphi\right)(I)=x^{*}(\varphi(I)),
$$

and, since $I$ and $x^{*}$ are arbitrary, we obtain that $f$ is $\mathcal{W M}$-integrable on $S$ and, for every $I \in \mathscr{S}$,

$$
\text { (WM) } \int_{I} f=\varphi(I) \text {. }
$$

(iii) $\Rightarrow$ (i). Let $v: \mathcal{L} \rightarrow X$ be a $\lambda$-continuous countable additive measure of bounded variation. We define a function $\varphi \in \Phi$ as follows:

$$
\varphi(I)=v(I), \quad I \in \mathscr{S} .
$$

Since $v$ is $\lambda$-continuous, its variation $|v|$ is also $\lambda$-continuous, and since $|v|$ is a bounded measure we obtain by [6, Theorem 6.11] that to a given $\varepsilon>0$ there exists $\eta>0$ such that, for every $E \in \mathcal{L}$,

$$
\lambda(E)<\eta \Rightarrow|v|(E)<\varepsilon .
$$

Let $D$ be a finite collection of nonoverlapping intervals in $\mathscr{S}$ such that

$$
\bigcup_{I \in D} \lambda(I)<\eta
$$

Then

$$
\sum_{I \in D}\|\varphi(I)\|=\sum_{I \in D}\|v(I)\| \leq \sum_{I \in D}|v|(I)=|v|\left(\bigcup_{I \in D} I\right)<\varepsilon .
$$

This means that $\varphi$ is $s A C$ and therefore we obtain by Lemma 3.1 that $V_{\varphi}^{\mathcal{M}} \ll \lambda$. Hence, by (iii), there exists a function $f: S \rightarrow X$ such that $f$ is $\mathcal{W} \mathcal{M}$-integrable on $S$ and, for every $I \in \mathscr{S}$,

$$
v(I)=\varphi(I)=(\mathrm{WM}) \int_{I} f .
$$


Now we will show that $f$ is Pettis integrable on $S$. Since each real-valued McShane integrable function is Lebesgue integrable we obtain that for each $x^{*} \in X^{*}$ the real function $x^{*} f$ is Lebesgue integrable. Thus it remains to prove that for every $E \in \mathcal{L}$ there exists $x_{E} \in X$ such that, for every $x^{*} \in X^{*}$,

$$
x^{*}\left(x_{E}\right)=(L) \int_{E} x^{*} f,
$$

where $(L) \int_{E} x^{*} f$ denotes the Lebesgue integral of $x^{*} f$ over $E$.

First we consider an open subinterval $I$ of $S$. We denote by $\bar{I}$ the closure of $I$ in $S$. Note that

$$
\text { (L) } \int_{I} x^{*} f=(L) \int_{\bar{I}} x^{*} f=x^{*}\left((\mathrm{WM}) \int_{\bar{I}} f\right)=x^{*}(v(\bar{I})) .
$$

Thus we have $x_{I}=v(\bar{I})=v(I)$.

Secondly, let $G$ be an open subset of $S$. There exists a sequence $\left(I_{k}\right)$ of pairwise disjoint open subintervals of $S$ such that $G=\bigcup_{k=1}^{\infty} I_{k}$. Then

$$
\text { (L) } \begin{aligned}
\int_{G} x^{*} f & =(L) \int_{\bigcup_{k=1}^{\infty} I_{k}} x^{*} f=\sum_{k=1}^{\infty}(L) \int_{I_{k}} x^{*} f \\
& =\sum_{k=1}^{\infty} x^{*}\left(x_{I_{k}}\right)=\sum_{k=1}^{\infty} x^{*}\left(v\left(I_{k}\right)\right)=\lim _{n \rightarrow \infty} \sum_{k=1}^{n} x^{*}\left(v\left(I_{k}\right)\right) \\
& =\lim _{n \rightarrow \infty} x^{*}\left(\sum_{k=1}^{n} v\left(I_{k}\right)\right)=x^{*}\left(v\left(\bigcup_{k=1}^{\infty} I_{k}\right)\right)=x^{*}(v(G)) .
\end{aligned}
$$

Hence, $x_{G}=v(G)$.

Finally, we consider a measurable set $E \in \mathcal{L}$. There exists a sequence $\left(G_{n}\right)$ of open subsets of $S$ such that, for every $n \in \mathbb{N}$,

$$
E \subset G_{n}, \quad G_{n+1} \subset G_{n}
$$

and $\lambda\left(G_{\delta} \backslash E\right)=0$, where $G_{\delta}=\bigcap_{n=1}^{\infty} G_{n}$. Since $v$ is a $\lambda$-continuous countable additive measure and $\lim _{n \rightarrow \infty} \lambda\left(G_{n}\right)=\lambda\left(G_{\delta}\right)$ we obtain by [2, Theorem I.2.1] that $\lim _{n \rightarrow \infty} v\left(G_{n}\right)=v\left(G_{\delta}\right)$. Therefore,

$$
\text { (L) } \begin{aligned}
\int_{E} x^{*} f & =(L) \int_{G_{\delta}} x^{*} f-(L) \int_{G_{\delta} \backslash E} x^{*} f=(L) \int_{G_{\delta}} x^{*} f \\
& =\lim _{n \rightarrow \infty}(L) \int_{G_{n}} x^{*} f=\lim _{n \rightarrow \infty} x^{*}\left(x_{G_{n}}\right)=\lim _{n \rightarrow \infty} x^{*}\left(v\left(G_{n}\right)\right)=x^{*}\left(v\left(G_{\delta}\right)\right),
\end{aligned}
$$

and so $x_{E}=v\left(G_{\delta}\right)=v(E)$.

Consequently the function $f$ is Pettis integrable and, for every $E \in \mathcal{L}$,

$$
v(E)=(P) \int_{E} f .
$$

This proves that $X$ has the WRNP. 


\section{References}

[1] B. Bongiorno, L. Di Piazza and K. Musial, 'A characterization of the Weak Radon-Nikodym property by finitely additive interval functions', Bull. Aust. Math. Soc. 80 (2009), 476-485.

[2] J. Diestel and J. J. Uhl, Vector Measures, Mathematical Surveys, 15 (American Mathematical Society, Providence, RI, 1977).

[3] L. Di Piazza, 'Variational measures in the theory of the integration in $\mathbb{R}^{n}$ ', Czechoslovak Math. J. 51(126) (2001), 95-110.

[4] T. Y. Lee, 'A full descriptive definition of the Henstock-Kurzweil integral in the Euclidean space', Proc. Lond. Math. Soc. (3) 87 (2003), 677-700.

[5] W. F. Pfeffer, Derivation and Integration (Cambridge University Press, Cambridge, 2001).

[6] W. Rudin, Real and Complex Analysis, 2nd edn (McGraw-Hill, New York, NY, 1974).

[7] Š. Schwabik and G. Ye, Topics in Banach Space Integration, Series in Real Analysis, 10 (World Scientific, Hackensack, NJ, 2005).

[8] V. A. Skvortsov and A. P. Solodov, 'A variational integral for Banach-valued functions', Real Anal. Exchange 24 (1998/9), 799-806.

[9] B. S. Thomson, 'Derivates of interval functions', Mem. Amer. Math. Soc. (1991), 452.

[10] B. S. Thomson, 'Differentiation', in: Handbook of Measure Theory (ed. E. Pap) (Elsevier Science B.V., 2002), pp. 179-247.

[11] G. Ye and S. Schwabik, 'The McShane and the weak McShane integrals of Banach space-valued functions defined on $\mathbb{R}^{m}$, Math. Notes (Miskolc) 2 (2001), 127-136.

\section{SOKOL BUSH KALIAJ, Science Natural Faculty, Mathematics Department, University of Elbasan, Elbasan, Albania \\ e-mail: sokol_bush@yahoo.co.uk}

